\title{
Proposta de instrumento de atenção primária à saúde da pessoa com deficiência
}

\author{
Proposal for a primary instrument the attention to the health of people with disabilities
}

\author{
Gabriela dos Santos Vera Ortiz ${ }^{1}$, Lívia Keismanas de Ávila² ${ }^{2}$ Evelyn Fabiana Costa ${ }^{3}$
}

\section{Resumo}

Objetivo: Elaborar um instrumento de identificação e acompanhamento da pessoa com deficiência. Método: Pesquisa retrospectiva, a partir de 25 "Folhas de Controle", aplicadas no desenvolvimento do PET-PcD às famílias com portadores de deficiência cadastradas na Unidade Básica de Saúde. A análise foi baseada no referencial teórico da Taxonomia de Necessidades de Saúde de Matsumoto. Resultados: No que se refere a necessidade de ter boas condições de vida, $30 \%$ dos deficientes físico/motor participam de atividades sociais, $50 \%$ dos deficientes visuais e $33,4 \%$ dos deficientes mental/intelectual. Sobre o acesso e uso de tecnologias, nos deficientes físicos, $80 \%$ utilizam algum tipo de tecnologia; dos deficientes visuais, $75 \%$ usam bengala branca; dos deficientes mentais/intelectuais, 33,4\%, utilizam cadeira de rodas. Sobre a necessidade de ser acolhido e ter vínculo com um profissional ou equipe, $90 \%$ dos deficientes físicos/motores, $75 \%$ dos deficientes visuais e $100 \%$ deficientes mentais/ intelectuais e deficientes múltiplos tem acompanhamento médico. Sobre o desenvolvimento de autonomia, 30\% dos deficientes físicos recebem cuidados, dos deficientes visuais $50 \%$ recebem, dos deficientes mental/intelectual 100\% têm cuidado de familiar e os deficientes múltiplos 87,5\%. Foi assim criado um instrumento para a atenção primária, este foi diferenciado por cores e separados por campos, conforme os grupos de necessidades. Conclusão: O desenvolvimento de uma proposta de instrumento para auxiliar na identificação e acompanhamento do deficiente nos serviços de atenção

1. Acadêmica da Faculdade de Ciências Médicas da Santa Casa de São Paulo. $8^{\circ}$ Semestre do Curso de Graduação em Enfermagem

2. Professora Adjunta da Faculdade de Ciências Médicas da Santa Casa de São Paulo. Curso de Graduação em Enfermagem

3. Preceptor-PET-Saúde Programa de Educação pelo Trabalho para a Saúde

Trabalho realizado: Faculdade de Ciências Médicas da Santa Casa de São Paulo - Curso de Graduação em Enfermagem Endereço para correspondência: Gabriela dos Santos Vera Ortiz. Faculdade de Ciências Médicas da Santa Casa de São Paulo. Curso de Graduação em Enfermagem. Rua Doutor Cesário Motta Jr, 61 - Vila Buarque -01221-020 - São Paulo, SP - Brasil. E-mail: gabiorthiz@hotmail.com primária contribui para a integralidade da assistência e possibilita o planejamento e desenvolvimento de ações.

Descritores: Atenção primária à saúde, Pessoas com deficiência

\begin{abstract}
Objective: The objective was to build an instrument of identification and follow up of a person with deficiency. Method: Retrospective research. The data source were the 25 "Control Forms", applied to the relatives of the deficient listed on the Blue and Green Teams of a Basic Health Unit during development of the PET-PCD. The data was analyzed following the Matsumoto's Heath Needs Taxonomy theoretical referential. Results and Discussion: About the needs of a good life conditions: physical/motor deficients: $30 \%$ participate of community activities and $70 \%$ do not participate; visual deficients: $50 \%$ participate and $50 \%$ do not; mental/intelectual deficients: $33,4 \%$ participate and $66,6 \%$ do not. About the needs of use of hard technology, $80 \%$ of the physical deficients do use, which $40 \%$ use a wheelchair, 30\% crutches and 10\% walker; of the visual deficients $75 \%$ use the white stick; 33,4\% of the mental/ intelectual deficients use wheelchair. About the need of a professional following, $90 \%$ of the physical/motor, $75 \%$ of the visual and $100 \%$ of the mental/intelectual deficients reported regular medical accompaniment. Hence, it was built an instrument for primary attention, which was divided into collors and fields, according tho the groups of needs. Conclusion: the development of an instrument to help in the identification and following of a defficient in the primary attention service do contribute to the assistance integrallity and allows planning and development of actions.
\end{abstract}

Keywords: Primary health care, Disabled person

\section{Introdução}

Em 2013, com o objetivo de promover a integração ensino-serviço para a formulação da rede de cuidados à pessoa com deficiência, a Faculdade de Ciências Médicas da Santa Casa de São Paulo foi 
contemplada, pelo Ministério da Saúde, com bolsas tutoriais para o desenvolvimento de projetos de pesquisa e intervenções relacionadas ao Programa Educação pelo Trabalho-Pessoa com Deficiência $(\mathrm{PETPCD})^{(1)}$. As atividades foram desenvolvidas nos diferentes serviços de saúde pertencentes à Coordenadoria Regional de Saúde-Sé, do município de São Paulo, dentre eles a Unidade Básica de Saúde Dr. Otacílio Rodovalho - Bom Retiro ${ }^{(2)}$. Neste serviço foram realizadas ações com os agentes de saúde na intenção de sensibilizá-los sobre a definição de deficiência, e a realização de visitas domiciliares às famílias com pessoas com deficiência, a fim de aprimorar a identificação, cadastro e acompanhamento desses usuários. O desenvolvimento desta atividade possibilitou reconhecer as diferentes percepções dos agentes comunitários de saúde em relação ao usuário com deficiência.

A Estratégia Saúde da Família (ESF) se caracteriza na assistência da Atenção Primária a Saúde um importante movimento de reorientação do modelo de atenção à saúde em nosso país. Caracteriza-se pela integração e pela organização das atividades em um território definido com enfoque principal na família, no contexto do ambiente em que vive, e numa maior proximidade com a comunidade ${ }^{(3)}$.

A ESF é composta por equipe multiprofissional que possui, no mínimo, um médico generalista ou especialista em saúde da família ou médico de família e comunidade, um enfermeiro generalista ou especialista em saúde da família, um ou dois auxiliares ou técnicos de enfermagem e cinco a seis agentes comunitários de saúde (ACS) ${ }^{(3)}$.

A atividade específica do ACS é identificar áreas e situações de risco individual e coletivo, encaminhar as pessoas aos serviços de saúde sempre que necessário, orientar as pessoas, de acordo com as instruções da equipe de saúde, acompanhar a situação de saúde das pessoas, para ajudá-las a conseguir bons resultados ${ }^{(4)}$.

Os ACS são os primeiros a realizarem um contato direto de identificação dos portadores de deficiência e, portanto, devem ser bem orientados e treinados para realizarem essa triagem, devem ser capacitados; para interagir com esse grupo específico nos serviços de saúde ${ }^{(4)}$.

A atuação do ACS no acompanhamento à saúde da pessoa com deficiência se concretiza na realização de visitas diárias a moradores da região de abrangência de sua UBS, na busca ativa de deficientes e no levantamento de problemas, que são encaminhados a equipe multidisciplinar para ser gerada resoluções. Para nortear essa prática em serviço, o "Guia Prático do ACS" conceitua e classifica as pessoas com deficiências como aquelas que têm "impedimentos de longo prazo de natureza física, mental, intelectual ou sensorial que, ao encontrarem com diversas barreiras, podem ter dificuldades em sua participação plena e efetiva na sociedade em igualdade de condições com as demais pessoas". As deficiências são classificadas em: Deficiência Auditiva; Deficiência Física (mencionam-se casos de pessoas acamadas pós AVC (Acidente Vascular Cerebral) e Paralisia Cerebral; Deficiência Visual; Deficiência Intelectual ${ }^{(4)}$.

De acordo com os fatos mencionados e com o desenvolvimento do PET/Pró-Saúde na Coordenadoria Regional de Saúde Centro, na UBS Dr. Otacílio Rodovalho - Bom Retiro foram realizadas ações com os agentes de saúde na intenção de sensibilizá-los sobre a definição de deficiência a fim de aprimorar a identificação, cadastro e acompanhamento dos deficientes. Essas atividades se desenvolveram em diferentes etapas:

$1^{\mathrm{a}}$ - Iniciou pela identificação das pessoas com deficiência residentes na área de abrangência das Equipes Verde a Azul da Estratégia Saúde da Família, áreas essas compreendidas pelas proximidades da região do Bom Retiro e Estação da Luz. Esta ação foi realizada a partir do levantamento das informações contidas no Sistema de Informação da Atenção Básica (SIAB) e por meio da Ficha A (Ficha de Identificação do Usuário), instrumento utilizado pelos Agentes Comunitários de Saúde no cadastro dos usuários.

$2^{\mathrm{a}}$ - Identificado as famílias que continham membro com deficiência, procedeu-se a consulta ao prontuário com a finalidade de reconhecer esse usuário, assim como suas necessidades, e os atendimentos realizados pelos ACS e equipe multidisciplinar.

$3^{\mathrm{a}}$ - $\quad$ Às famílias incluídas na segunda etapa foram realizadas visitas domiciliares junto aos ACS da área de abrangência para o preenchimento da "Folhas de Controle" anexo I, com a finalidade de caracterizar e identificar as necessidades em saúde da pessoa com deficiência, sob a ótica do profissional de saúde (enfermeiro, fonoaudiólogo e equipe do Núcleo de Apoia à Saúde da Família - NASF).

Destacamos que a "Folha de Controle" foi elaborada com base nos critérios da Classificação Internacional de Funcionalidade (CIF), considerando que, "toda perda ou anormalidade de uma estrutura ou função psicológica, fisiológica ou anatômica que gere incapacidade para o desempenho de atividade, dentro do padrão considerado normal para o ser humano, é classificado como deficiência, essas podem ser divididas em: a) deficiência física; b) deficiência auditiva; c) deficiência visual; d) deficiência mental" (5). $4^{a}$ - Com o intuito de criar um saber coletivo acer- 
ca de alguns pontos importantes da rotina de vida das pessoas portadoras de alguma deficiência, foi realizada uma sensibilização com os ACS. Para isso, foram expostas frases que retratam mitos, verdades e questionamentos sobre a temática deficiência, nos ambientes de convívio comum como: sala de reunião, cozinha, consultórios, setor administrativo. Posteriormente, esses panfletos com mensagens foram discutidos em quatro oficinas educativas.

A partir desse contexto este estudo pretende contribuir para a elaboração de estratégias que reconheçam esses indivíduos, e suas necessidades em saúde, suas especificidades e demandas por meio de um instrumento de identificação e acompanhamento da pessoa com deficiência.

\section{Método}

Tratou-se de uma pesquisa retrospectiva, em que o estudo é desenhado para explorar fatos do passado, utiliza-se um ponto do passado a fim de conduzir a pesquisa até o momento presente, pela análise documental ${ }^{(6)}$. O presente estudo foi realizado a partir de dados coletados na UBS Bom Retiro - Dr. Octávio Augusto Rodovalho localizada na região central do município de São Paulo, sob supervisão da Coordenadoria Regional de Saúde Centro, parceira no desenvolvimento do Programa Educação pelo Trabalho (PET) da Faculdade de Ciências Médicas da Santa Casa de São Paulo. A fonte de dados foi constituída pelas 25 "Folhas de Controle", aplicadas durante o desenvolvimento do Programa Educação pelo Trabalho (Pet- PcD). O projeto foi submetido à Comissão Científica do Curso de Enfermagem da FCMSCSP, em seguida ao Comitê de Ética em Pesquisa com Seres Humanos da Santa Casa de São Paulo e Secretaria Municipal de Saúde de São Paulo, conforme Resolução 466/2012, parecer n ${ }^{\circ}$ 1.564.798. As informações existentes nas "Folhas de Controle" foram agrupadas em planilha elaborada no Programa Microsoft Excel ${ }^{\circledR}$ e analisados seguindo o referencial teórico da Taxonomia de Necessidades de Saúde de Matsumoto (1999) ${ }^{(7)}$.

\section{Resultados e Discussão}

De acordo com a Taxonomia de Necessidades de Saúde e Matsumoto, as necessidades de saúde são compreendidas, de forma bastante completa, em uma divisão organizada em quatro grandes conjuntos de necessidades.

O primeiro conjunto, diz respeito a se ter "boas condições de vida", o que integra, necessidade de boa moradia, alimentação, transporte, lazer, meio ambiente adequado, viver em processos sociais de inclusão, ter boas condições de trabalho, outras atividades que não sejam o trabalho e a presença de necessidades morais e espirituais ${ }^{(7)}$.

Considerando este primeiro conjunto de necessidades de saúde, em relação aos deficientes físicos / motor, estes em sua maior parte, não possuem um momento para o lazer, uma vez que $70 \%$, dos entrevistados não participam de nenhuma atividade dentro da comunidade. No caso dos deficientes visuais, $50 \%$, não participam de atividades dentro da comunidade, outros $66,6 \%$ de deficientes mentais/ intelectuais também não participam dessas atividades. Em relação aos deficientes múltiplos, 62,5 não participam.

Ao serem interrogados sobre o uso do transporte público, os dados obtidos conferem que $70 \%$ dos deficientes físicos não fazem uso, muitos dizem não ter tantos transportes adaptados, optando por táxi, ou carro particular. Dos deficientes visuais houve uma equivalência de $50 \%$ fazem uso de transporte público, estes utilizam o metrô, dizendo que o piso podotátil facilita a transição nesses locais, outros $50 \%$ não fazem uso, por utilizarem táxi.

A frequência dos deficientes múltiplos que não fazem uso do transporte público é ainda maior são $75 \%$, sendo que $1 / 3$ necessita de ambulância para sua remoção, devido as suas dificuldades, outros $25 \%$ fazem uso, porém apresentam adaptações, como cadeiras adaptadas que facilitam a transição nas ruas. Dos deficientes mentais $66,6 \%$, fazem uso de transporte público, e 33,3\%, não fazem uso.

Este questionamento nos permitiu identificar as dificuldades diárias da locomoção dessas pessoas para os atendimentos de saúde, ou até mesmo as casas de familiares, ou para momentos de lazer. As barreiras arquitetônicas impostas às pessoas com limitações, como os deficientes, são barreiras que impedem o exercício do mais básico dos direitos de qualquer cidadão, o de deslocar-se livremente. A presença de transporte inadequado, sem adaptação necessária para esse público, impede a inclusão desse indivíduo nos meios sociais, negando o direito de ir e $\operatorname{vir}^{(8)}$.

Em relação a alimentação dos deficientes físicos / motores pesquisados, $80 \%$, recebem dieta geral, $10 \%$ dieta hipossódica, outros $10 \%$ dieta pastosa, estas duas últimas devido comorbidade associada. Já nos deficientes visuais $75 \%$ fazem uso de dieta geral, e $25 \%$ dieta hipossódica, $100 \%$ dos mentais/intelectuais recebem dieta geral, $37,5 \%$ dos deficientes múltiplos, recebem dieta geral, 25,0\% dieta triturada, $12,5 \%$ dieta pastosa, e outras $12,5 \%$ dieta hipossódica. Nas "Folhas de Controle" foi identificada a associação de dietas relacionadas às comorbidades, como no caso da dieta hipossódica, que está associada a hipertensão arterial. 
A alimentação é um marcador de saúde necessário para garantir a qualidade de vida do indivíduo, uma alimentação equilibrada gera benefícios físicos indispensáveis, o conceito ideal da alimentação, portanto, é definido pelos profissionais como uma condição alimentar que se adeque as necessidades nutricionais do indivíduo, como no caso da pessoa hipertenso que opta por uma dieta pobre em sódio ${ }^{(9)}$.

O segundo conjunto de necessidades de saúde se relaciona a ter acesso e poder utilizar toda tecnologia de saúde capaz de melhorar e prolongar a vida, seja ela tecnologia dura, referente ao uso de equipamentos tecnológicos, e a utilização de estrutura organizacional como encontrado na rede de cuidados, nos níveis de atenção à saúde, ou o uso de tecnologia leve o que se diz respeito a produção de vínculo entre equipe e usuário, assim como as informações e educação

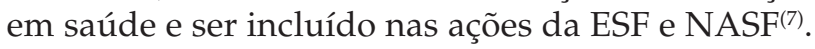

No que se refere ao uso da tecnologia dura, dos deficientes físicos entrevistados, $70 \%$, diz ter passado por algum procedimento cirúrgico, dos deficientes visuais $100 \%$, revelam ter passado por cirurgia, $66,6 \%$ dos deficientes mentais/intelectuais passaram por cirurgias, e outros $33,3 \%$, referem não ter passado, $87,5 \%$ dos deficientes múltiplos passaram por cirurgia. Essa questão nos demonstrou que uma grande parcela dos deficientes depende da incorporação de tecnologia dura.

No questionamento referente ao uso de aparelho ou equipamento devido à deficiência, percebemos que dos deficientes físicos, $80 \%$ faz uso, destes $40 \%$ utilizam cadeira de rodas, $30 \%$ muletas, $10 \%$ andador. Os números coletados demonstraram que entre os deficientes visuais, $75 \%$ fazem uso de bengala branca instrumento básico de locomoção e $25 \%$ não fazem uso de nenhum aparelho; dos deficientes mentais/intelectuais, 33,3\% utiliza cadeira de rodas; e dos deficientes múltiplos $87,5 \%$ utilizam aparelho para deficiência, destes $62,5 \%$ faz uso de cadeira de rodas, $12,5 \%$ uso de bengala, $12,5 \%$ uso de aparelho auditivo.

Matsumoto (1999)(7) cita o uso da tecnologia dura como uma importante ação especializada e, sempre que for necessário, o usuário poderá e deverá consumir serviços de saúde (saberes, equipamentos e práticas) que possam impactar e qualificar seu modo de vida.

O terceiro conjunto, segundo a taxonomia, é a necessidade de "ser acolhido e ter vínculo com um profissional ou equipe". "A arte da fala e da escuta são os principais instrumentos para isso" (7). Saber a quem se direcionar e ainda ter um "rosto" na equipe a quem possa confiar e direcionar suas questões, esse papel muitas vezes é preenchido pelo ACS, ou por qualquer outro profissional que crie um elo de confiança com o cliente.
O terceiro grupo de necessidades em saúde se refere ao estreitamento das relações entre o profissional e o usuário, no qual se destaca a afirmativa "ser acolhido e ter vínculo com um profissional ou equipe". Sobre este grupo, os dados coletados demonstraram que entre os deficientes físicos/motores $90 \%$ relatam ter que fazer acompanhamento médico regular, $10 \%$; dizem não necessitar; dos deficientes visuais $75 \%$, fazem acompanhamento, em sua maioria pela comorbidade que o levou a deficiência, neste caso o Diabetes Mellitus, 25\% não fazem acompanhamento. Algo interessante foi em relação aos deficientes mentais/intelectuais e deficientes múltiplos, que 100\% disseram fazer acompanhamento médico regular, este fato se dá pela demanda de cuidados que estes clientes necessitam, em sua maioria fazem uso de fralda descartável, e tem acompanhamento com equipe especializada, como Fisioterapeutas, Fonoaudiólogas e médicos especialistas.

Isso se refere a ideia do vínculo que se cria entre profissional e paciente, isso gera uma maior eficácia, no acompanhamento, a valorização e a construção de espaços propícios à produção de sujeitos autônomos que podem acessar e serem recebidos e bem acolhidos em qualquer serviço de saúde que necessitarem ${ }^{(7)}$.

Um quarto, e último conjunto de necessidades, diz respeito a necessidade de cada pessoa ter graus crescentes de autonomia no seu modo de viver. A educação em saúde é apenas parte do processo de construção da autonomia de cada pessoa e isso implica seu modo de viver, incluindo a busca pela satisfação de suas necessidades ${ }^{(7)}$.

Neste último conjunto tem-se a autonomia definida, muitas vezes, pelo cuidado dispendido pelo familiar ou cuidador, nesta relação identificamos que apenas 30\% dos deficientes físicos referiram receber cuidados, dos deficientes visuais, $50 \%$ recebem, dos deficientes mental/intelectual, $100 \%$ recebem cuidado de familiar e os deficientes múltiplos $87,5 \%$ recebem cuidados.

Em relação à identificação da dificuldade de locomoção dentro da própria casa e a necessidade de adaptação. Os dados demonstraram que entre os deficientes físicos, 30\% têm dificuldade de locomoção dentro da própria casa, e ainda diz que $50 \%$ desses deficientes possuem adaptações e $50 \%$ não possuem adaptações em sua casa. Dos deficientes visuais, 50\% têm dificuldade e apenas 25\% têm adaptações em sua casa, como em um dos casos que possui barras de segurança no banheiro, o que difere dos outros $75 \%$ que não possuem adaptações e referem conviver relativamente bem com isso. Os deficientes mental/ intelectual, 33,4\% têm dificuldade de locomoção na residência e por isso possuem adaptações em sua residência dos deficientes múltiplos, 50\%, têm 
dificuldade de locomoção, o que demonstra que dos deficientes múltiplos os que não possuem adaptações tem associado dificuldades de locomoção. Os demais que relatam dificuldades de locomoção referiram que isso acontece devido, principalmente, a falta de acessibilidade das moradias ( $8 \%$ dos casos), em que os deficientes precisavam ser içados pelos familiares, por não ter elevador em seu prédio. E, outros aprenderam a conviver coma adversidade, pois não possuem adaptações em seu domicílio.

Existem vários fatores que dificultam a concretização das modificações domiciliares, entre eles, a falta de conhecimento desta necessidade pelo familiar e ou cuidador, o custo e a prestação de serviços que se adequem as necessidades dos deficientes, ou o ambiente físico de moradia que muitas vezes não permite tais alterações. Além de barreiras arquitetônicas no domicílio, como degraus no acesso a casa, portas estreitas, tapetes não antiderrapantes, entre outros ${ }^{(10)}$.

\section{Conclusão}

A partir da análise dos dados das "Folhas de Controle" conclui-se que a elaboração de um instrumento de identificação e acompanhamento da pessoa com deficiência possibilita uma compreensão ampliada das diferentes necessidades em saúde do usuário.

No âmbito das ações relacionadas a inserção do usuário em espaços de lazer, questionamentos como "Os centros de lazer e convivência possuem adaptação de acessibilidade física e visual para esse público? Você tem alguém que pode te acompanhar em suas atividades de lazer?", contribuem para identificar o que impede a inclusão social deste público e se isso se limita apenas as barreiras arquitetônicas impostas.

Em relação ao uso de transporte público, outras informações relevantes seriam: - O ônibus que utiliza é adaptado? - O ponto de ônibus é próximo a sua residência? - Tem necessidade de remoção por ambulância para chegar aos serviços de saúde?

A avaliação e acompanhamento da condição nutricional também é fundamental, pois implica na manutenção de um estado de saúde, assim é fundamental questionamentos como: - Possui comorbidade que é necessário adequar a dieta?; - Quantas refeições realiza por dia? Você precisa de auxílio para alimentar-se? Com isso durante as atividades desempenhadas na atenção básica é possível orientar e promover um aporte nutricional proporcional as demandas.

Além disso, se faz importante também sabermos as condições de uso dos aparelhos/equipamentos utilizados pelos deficientes, se há necessidade de troca dos pneus, estofado, dos cadeirantes ou troca de muletas e andadores por mau uso. E ainda, sobre o uso de tecnologia dura, a seguinte interrogação se faz necessária: - Quais cirurgias realizadas? Podemos assim identificar outras necessidades decorrentes de tais procedimentos como, por exemplo, o uso de algum dispositivo, como bolsa de colostomia, cateter vesical de demora e outros.

Entende-se que sobre o estreitamento de relação entre profissional e usuário, as questões que deveriam ser respondidas são: - Qual a frequência da visita do Médico da Família? - Qual especialidade necessita? - Já recebeu visita de quais especialidades? Isso se refere a necessidade de ter acolhimento nos serviços de saúde sempre que precisar, ter a saúde acompanhada por uma equipe de referência, construir vínculo com os profissionais.

Relacionado à autonomia do usuário foram mantidas as questões: Possui alguma dificuldade de locomoção dentro da própria casa? Recebe algum cuidado de familiares e ou cuidador particular? Necessita de auxílio para realizar atividades de vida diária?

Assim, o agrupamento dessas informações a partir das necessidades de saúde, permitiu o desenvolvimento do instrumento diferenciado por cores para melhor visualização dos questionamentos que respondem a perspectiva de saúde e separados por campos, conforme anexo, que contribui para a integralidade da assistência e possibilita o planejamento e desenvolvimento de ações que considerem o princípio da equidade como norteador do cuidado em saúde.

\section{Anexo}

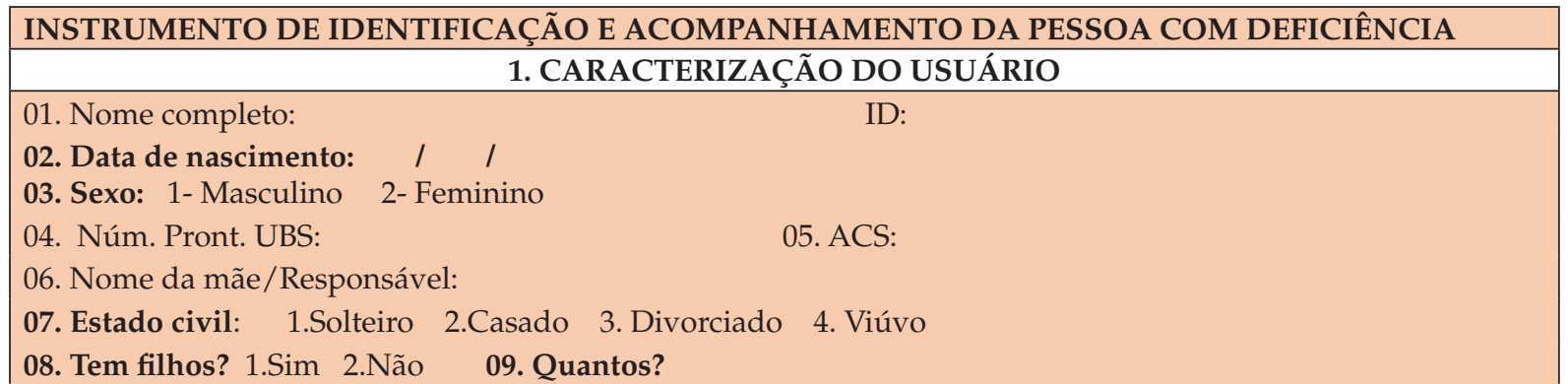


10. Grau de escolaridade:

1) Analfabeto

2) Alfabetizado

3) Ensino fundamental incompleto

4) Ensino fundamental completo

5) Ensino médio incompleto

6) Ensino médio completo

7) Superior incompleto

8) Superior completo

11. Tipo de deficiência: 1 . Física/Motora

2. Visual

3. Auditivo

4. Mental/Intelectual

5. Múltipla

12. Etiologia da Deficiência: 1 . Congênita 2. Adquirida

13. Deficiência: 1 . Temporária $\quad 2$. Permanente

14. Em caso de deficiência temporária, há quanto tempo?

\section{NECESSIDADES DE BOAS CONDIÇÕES DE VIDA}

\section{$\begin{array}{lll}\text { 15. Trabalha? 1. Sim 2. Não 16. Qual função exercida? } & \end{array}$}

17. Precisa de alguma adaptação no trabalho?

$\begin{array}{lll}\text { 18. Tipo de moradia: } 1 \text {. Casa } & \text { 2. Apartamento } & \text { 3. Outros }\end{array}$

19. Possui adaptação na residência?

20. Possui comorbidade que é necessário a adequação da dieta? 1. Sim 2. Não

21. Quantas refeições realiza por dia: 1.3 refeições

\section{3 a 5 refeições \\ 3. mais de 6 refeições diárias}

22. Qual Tipo de dieta: 1 . Geral
2. Triturado
3. Pastoso
4. Hipossódica

23. Quem realiza o preparo dos alimentos?

24. Quem oferece as refeições?

25. Faz uso de transporte público? 1. Sim 2.Não

25. O transporte que utiliza é adaptado? 1. Sim 2. Não

26. O ponto de ônibus é próximo a sua residência? 1. Sim 2.Não

27. Como se locomove: 1 . Ônibus

$$
\begin{aligned}
& \text { 2. Metrô/Trem } \\
& \text { 3. Táxi } \\
& \text { 4. Carro particular }
\end{aligned}
$$

28. Tem necessidade de remoção com ambulância para chegar aos serviços de saúde? 1 . Sim 2 . Não

29. Você tem alguém que pode te acompanhar em suas atividades de lazer? 1. Sim 2. Não

30. Participa de alguma forma, de atividades dentro da comunidade: 1 .Sim 2.Não

31.Os centros de lazer e convivência possuem adaptação de acessibilidade física e visual para esses públicos?

1. Sim 2.Não

32. O que costuma fazer para se divertir?

\section{NECESSIDADES DE USO DE TECNOLOGIA DURA}

\section{Já realizou algum procedimento cirúrgico? 1 . Sim 2. Não}

34. Qual procedimento cirúrgico?

35. Quais dispositivos/artigos de higiene necessita em seu dia a dia?

1. Fralda descartável

3. Bolsa de Colostomia

4. Sonda vesical de alívio

36. Aparelho devido deficiência? 1. Sim 2. Não

37. Como foi adquirido? 1. Comprado 2. Doado 


\section{Qual aparelho? \\ 1. Cadeira de rodas \\ 2. Muletas/Bengala \\ 3. Aparelho auditivo \\ 4. Bengala Branca \\ 5. Andador \\ 6. Outros, Qual?}

39. Quais condições de uso do aparelho? 1. Boa 2. Média 3. Ruim

40. Necessita de troca?

1. Estofado em caso de cadeira de rodas

2. Pneus em caso de cadeira de rodas

3. Muletas/ Bengala em mau uso

4. Andador em mau uso

5. Aparelho auditivo em más condições de uso

41. Necessita que encaminhe para algum serviço especializado?

1. Assistência técnica

2. CER (Centro Especializado de Reabilitação)

4. NECESSIDADE DE ESTREITAMENTO DE VÍNCULO PROFISSIONAL/USUÁRIO

42. Necessita de acompanhamento médico regular? 1.Sim 2. Não

43. Qual a frequência da visita do médico responsável?

1. Uma vez por mês

2. Trimestral

3. Semestral

4. Anual

44. Qual especialidade necessita?

45. Já recebeu visita de quais especialidades?

1. Enfermeiro

2. Fonoaudiologia

3. Psicólogo

4. Fisioterapeuta

5. Educador Físico

6. Nutricionista

7. Médico de família

5. NECESSIDADE DE DESENVOLVIMENTO DE AUTONOMIA

46. Possui alguma dificuldade de locomoção dentro da própria casa: 1 .Sim 2.Não

47. Recebe algum cuidado de familiares e ou cuidador particular? 1 . Sim 2. Não

48. Necessita de auxílio para realizar atividades de vida diária? 1 . Sim 2.Não

OBS:

\section{Referências}

1. Brasil. Ministério da Saúde. Departamento de Gestão da Educação na Saúde (DEGES). Secretaria de Gestão do Trabalho e da Educação da Saúde (SGTES). Pró-Saúde e PET-Saúde. Brasília: Ministério da Saúde; 2013.

2. Faculdade de Ciências Médicas da Santa Casa de São Paulo. Projeto referente ao PET-Saúde Redes 2013/2015. Disponível em: http://www.fcmsantacasasp.edu.br/images/Noticias/ petsaude/PET-SAUDE-REDES-DE-ATENCAO-A-PESSOA-COM-DEFICIENCIA.pdf [03 mar 2016]

3. São Paulo (Estado). Secretaria de Estado da Saúde. Atenção Básica. Disponível em: http://www.saude.sp.gov.br/ses/perfil/ gestor/atencao-basica/ [3 mar 2016].

4. Brasil. Ministério da Saúde. Secretaria de Atenção à Saúde.
Departamento de Atenção Básica. O trabalho do agente comunitário de saúde / Ministério da Saúde, Secretaria de Atenção à Saúde, departamento de Atenção Básica. - Brasília : Ministério da Saúde; 2009.

5. Organização Mundial da Saúde. Classificação Internacional da Funcionalidade Incapacidade e Saúde. Tradução Amélia Leitão. Portugal: Lisboa, 2004.

6. Fontenelles MJ, Simões MG, Farias S H, Fontenelles RGS. Metodologia da pesquisa ciêntífica: diretrizes para a elaboração de um protocolo de pesquisa. Disponível em: https://cienciassaude.medicina.ufg.br/up/150/o/Anexo_C8_NONAME.pdf>

7. Matsumoto NF. A operacionalização do PAS de uma Unidade Básica de Saúde no município de São Paulo, analisada sob o ponto de vista das necessidades de saúde. Dissertação (Mestrado). São Paulo: Escola de Enfermagem da Universidade de São Paulo; 1999. 
8. Siqueira FVC, Facchini LA, Silveira DS, Piccini RX, Thumé E, Tomasi E. Barreiras arquitetônicas a idosos e portadores de deficiência física: um estudo epidemiológico da estrutura físicas das unidades básicas de saúde em sete estados do Brasil. Ciênc Saúde Coletiva. 2009; 14(1):39-44.

9. Silva D O, Recine EGIG, Queiroz EFO. Concepções de profissionais de saúde sobre alimentação saudável. Cad Saúde Pública. 2002; 18(5):1367-77.
10. Garanhani MR, Alves JF, Fujisawa DS. Adaptação da pessoa após acidente vascular encefálico e seu cuidador: ambiente domiciliar, cadeira de rodas e de banho. Acta Fisiátrica. 2010; 17(4):164-8.

Trabalho recebido: $10 / 11 / 2016$

Trabalho aprovado: 19/05/2018 